\title{
PFC Sigma Cobalt-Chrome Total Knee Replacement: Early Outcomes Demonstrate No Significant Early Failures at the Three-Year Mark
}

\author{
Alexander M. Wood, Luke McIlwain, Calum H. Arthur, Stuart A. Aitken, Andre C. M. Keenan, \\ Phil Walmsley, Ivan Brenkel
}

Trauma and Orthopaedics, Victoria Hospital, NHS Fife, Kirkcaldy, UK.

Email: drsandywood@googlemail.com

Received November $6^{\text {th }}, 2013$; revised December $18^{\text {th }}, 2013$; accepted December $29^{\text {th }}, 2013$

Copyright (C) 2014 Alexander M. Wood et al. This is an open access article distributed under the Creative Commons Attribution License, which permits unrestricted use, distribution, and reproduction in any medium, provided the original work is properly cited. In accordance of the Creative Commons Attribution License all Copyrights @ 2014 are reserved for SCIRP and the owner of the intellectual property Alexander M. Wood et al. All Copyright (c) 2014 are guarded by law and by SCIRP as a guardian.

\section{ABSTRACT}

The PFC Sigma Cobalt Chrome Sigma (PFCSCC) was introduced in 2006, and represents further development of the PFC Sigma design aiming at reducing the problem of backside wear. To ensure that there were no significant early failures following the introduction of this knee system to our hospital in 2006, we prospectively identified all patients undergoing TKA with the PFCSCC over a one-year period. Clinical and demographic patient data, American Knee Society scores, Oxford Knee scores, SF-12 scores and radiographic data were recorded pre-operatively and at three-year post surgery. 233 patients underwent 249 primary knee arthroplasties with the PFCSCC. Seven patients (eight TKAs) died before the last review and eight cases were lost to follow up. Mean age was 66.7 (range 34 - 80) with $47.6 \%$ male. Mean follow-up days were 1109 (range 741 to 1591). 5 (2.2\%) were revised for infection with 1 revised for pain. The 3-year survival rate was $97.6 \%$ and $99.6 \%$ for aseptic failure. AKS $46.2(0$ - 95) was preoperatively $88.3(17-100)$ with 3 years $P<0.001$. OKS 39.0 (22 - 53) was preoperatively 22.6 (12 - 53) with 3 years $P<0.001 .17$ of the 219 who had $x$-rays $(8 \%)$ had radiolucent zones on $x$-ray. Our results demonstrate a good early aseptic survivorship of the PFCSCC at three years of $99.6 \%$, combined with a good functional and objective improvement in our patients in three years.

\section{KEYWORDS}

Arthroplasty; Knee; Outcomes

\section{Introduction}

Total knee arthroplasty is well established for relieving pain and improving function. The Press Fit Condylar (PFC) Sigma total knee arthroplasty (TKA) (Depuy, Johnson \& Johnson) is the most widely implanted knee prosthesis in England and Wales, and accounts for 36\% of all the TKAs performed in 2009 [1]. The PFC Sigma Cobalt Chrome (PFCSCC) TKA was introduced in 2006 and represents a design modification based upon the PFC Sigma.

The tibial tray for the PFCSCC TKA is made of a cobalt chrome alloy, whereas in the older PFC Sigma, the tray was made of titanium. The theoretical advantage of this design modification is that microscopically the co- balt chrome alloy is smoother than titanium and therefore less likely to produce backside wear of the polyethylene insert [2]. In addition, the PFCSCC polyethylene insert is exposed to a higher radiation dose than the previous PFC Sigma insert, theoretically resulting in an extended lifetime of the prosthesis. The locking mechanism between the insert and tibia tray has also been improved to minimize backside wear.

Minor changes in arthroplasty can lead to unexpected early catastrophic failure and change the survivorship of implants [3]. Many implants undergo minor changes and it is important to ensure that these changes do not cause early failure [4]. National joint registries [1] may not identify all minor changes and outcomes after a short 
period. Therefore, early clinical results in peer-reviewed journals represent an important method of informing surgeons about the initial survivorship and outcomes of newly introduced changes in the implant design and manufacture process [4]. We report the first early clinical, radiological and patient reported results for the PFCSCC TKA in 3 years, in order to allow comparison with published data on its predecessor, the PFC Sigma TKA.

\section{Methods}

Prospective data have been collected on all primary TKAs performed in our hospital since 1996. The PFCSCC (Depuy, Johnson \& Johnson) TKA was introduced in our hospital in February 2006. Between February 2006 and February 2007, 249 primary knee arthroplasties with the PFCSCC TKA were performed on a total patient cohort of 233. Sixteen patients underwent bilateral primary procedures. No other TKA implant designs were used during this time period.

Demographic and clinical data, American Knee Society (AKS), Oxford Knee (OKS) and Short Form-12 Items Survey (SF-12) scores were collected prospectively from all patients on admission and at follow up clinics at six months, 18 months and three years. At final review, the AKS score was calculated using the protocol described by Install et al. [5], and standard short leg anterioposterior and lateral radiographs were obtained. Radiographic measurements of varus and valgus angulation, flexion or extension of the tibial and femoral components were taken. Radiolucencies were sought at the bone/cement and cement/prosthesis interfaces of both components.

Eight different operating surgeons, either consultant grade or a trainee under direct supervision, performed the operations. All surgeons used the same instrumentation and patients underwent the same postoperative regimen. The operations were performed in a filtered air operating theatre with laminar flow. Waterproof single use gowns and drapes were used and both the surgeon and assistant were double gloved. A tourniquet was used routinely, and the femoral and tibial cuts were performed using intramedullary and extramedullary alignment, respectively. The patella was not routinely resurfaced, but this was carried out at the discretion of the operating surgeon when patella wear was severe. All patients had antibiotic prophylaxis with $1 \mathrm{~g}$ Ceftriaxone shortly before inflation of the tourniquet. Pre- and post-operative thromboembolic prophylaxis was administered in the form of using low molecular weight heparin and full-length graded elastic stockings [6]. Wounds were dressed with gauze, wool and crepe dressings. These dressings were removed on the first post-operative day and continuous passive mobilisation was commenced. Blood transfusion was only performed if the haemoglobin fell below $8.5 \mathrm{~g} / \mathrm{dl}$.

\section{Statistical Methods}

Continuous data were presented in terms of the mean and range (R). Mean values between groups (AKS scores, OKS, SF-12 scores) were compared using the $t$-test for two groups and the analysis of one-way variance (ANOVA) for three or more groups. A $p$-value of $<0.05$ determined statistical significance. Life tables were constructed and the cumulative survival rates were calculated. Endpoints chosen were "reoperation for any reason" and "component revision for aseptic loosening or mechanical failure". The Rothman method $[7,8]$ was used to calculate 95\% confidence intervals. A "worst case" survival analysis was also performed based on the assumption that all those lost to follow up had failed immediately after the time of their last appointment.

\section{Results}

Two hundred and forty-nine PFCSCC TKAs were performed on 233 patients, 122 (52.4\%) of whom were women. The indication for surgery was osteoarthritis in 226 (97.0\%) patients, rheumatoid arthritis in five (2.1\%) cases and avascular necrosis in two $(0.9 \%)$. The mean patient age was 66.7 years ( $\mathrm{R} 34-80 \mathrm{yr}$ ). The mean patient body weight was 83.2 kilograms (R 49 - $130 \mathrm{~kg}$ ) and the mean body mass index (BMI) was $30.0 \mathrm{~kg} / \mathrm{m}^{2}$ (R $20.7-40.1 \mathrm{~kg} / \mathrm{m}^{2}$ ).

Two hundred and forty-one TKAs in 226 patients were available for review at three years. The mean follow up was 1109 days (R 741 - 1591 days). The AKS score improved from 46.2 (R 0 - 95) pre-operatively to 88.3 (R 17 - 100) at final review ( $p<0.001, t$-test). The OKS (available for 203 patients) improved from 39.0 (R 22 53) pre-operatively to 27.6 (R 14 - 53) at three months, and 23.4 (R $12-53)$ at one year $(p<0.001$, ANOVA).

The patient mean pain score at admission was 11.7 (range 0 - 45) at 3 years: 39.5 (range 0 - 50) $(p<0.001)$. Function score mean at admission 43.5 (range -20 - 85). 59.5 (range $-20-90)$ at 3 -year follow up $(p<0.001)$.

Table 1 (Top) shows the mean patient reported SF-12 scores for 201 patients. By the three months post-operative time point, there was a statistically significant improvement in all SF-12 domains except the Mental Health score and General Health score. However, between the three months and one-year time points, the only SF-12 domains, which displayed a significant improvement, were the Physical Functioning and Pain scores.

Eight TKAs were lost to follow-up, five underwent revision for infection, and one was revised for persistent pain (Table 2). The three-year survival rate was calculated as $97.6 \%$ with "reoperation for any reason" (Figure 1) and $99.6 \%$ with "component revision for aseptic loo- 
Table 1. The mean SF-12 scores in each domain at the time of admission, and at three months, one year and three years. $p$-values for comparisons between selected mean values were calculated using $t$-tests. (Physical Functioning score [PF], Role Limitation Physical score [RP], Pain score [BP], General Health score [GH], Vitality score [VT], Social Functioning score [SF], Role Limitation Emotional score [RE] and Mental Health score [MH]).

\begin{tabular}{ccccccccc}
\hline \multirow{2}{*}{ SF-12 Domains } & \multirow{2}{*}{ Admission } & \multirow{2}{*}{ 3 Months } & 1 Year & 3 Years & Admission versus 3 & \multicolumn{3}{c}{ months versus 1 1 year versus 3 } \\
\hline PF & 6.2 & 49.3 & 55.7 & 51.4 & $<0.001$ & $<$ & 0.007 & 0.09 \\
RP & 3.9 & 44.9 & 48.6 & 48.6 & $<0.001$ & 0.28 & 0.51 \\
BP & 36.4 & 62.5 & 74.5 & 70.8 & $<0.001$ & $<0.001$ & 0.25 \\
GH & 68.4 & 66.4 & 65.0 & 60.3 & 0.38 & 0.24 & 0.020 \\
VT & 45.5 & 52.9 & 56.2 & 53.2 & $<0.001$ & 0.07 & 0.11 \\
SF & 52.4 & 74.9 & 77.5 & 76.1 & $<0.001$ & 0.30 & 0.69 \\
RE & 96.1 & 76.1 & 79.0 & 75.4 & $<0.001$ & 0.34 & 0.51 \\
MH & 69.8 & 72.3 & 74.9 & 72.6 & 0.12 & 0.10 & 0.13 \\
\hline
\end{tabular}

Table 2. The life table calculation for survival of the PFC Sigma Cobalt Chrome TKA, with chosen endpoints "reoperation for any reason", "component revision for aseptic loosening or mechanical failure" and "worse case" survival where all the knees lost to follow-up were assumed to have failed. (*Number of knee prosthesis implanted).

\begin{tabular}{ccccccccccc}
\hline Year & $\begin{array}{c}\text { No.*at } \\
\text { start }\end{array}$ & Death & $\begin{array}{c}\text { Lost to } \\
\text { follow up }\end{array}$ & Failure & No. at risk & $\begin{array}{c}\text { Annual } \\
\text { failure rate }\end{array}$ & $\begin{array}{c}\text { Annual } \\
\text { survival rate }\end{array}$ & $\begin{array}{c}\text { Cumulative } \\
\text { survival }\end{array}$ & $\begin{array}{c}\text { Cumulative worst } \\
\text { case survival }\end{array}$ & $\begin{array}{c}\text { Survival with } \\
\text { reoperation for } \\
\text { aseptic failure \# }\end{array}$ \\
\hline 1 & 249 & 1 & 3 & 6 & 247 & $2.4 \%$ & $97.6 \%$ & $97.6 \%$ & $96.4 \%$ & $99.6 \%$ \\
2 & 239 & 2 & 0 & 0 & 238 & $0.0 \%$ & $100.0 \%$ & $97.6 \%$ & $96.4 \%$ & $99.6 \%$ \\
3 & 236 & 5 & 5 & 0 & 231 & $0.0 \%$ & $100.0 \%$ & $97.6 \%$ & $94.3 \%$ & $99.6 \%$ \\
\hline
\end{tabular}

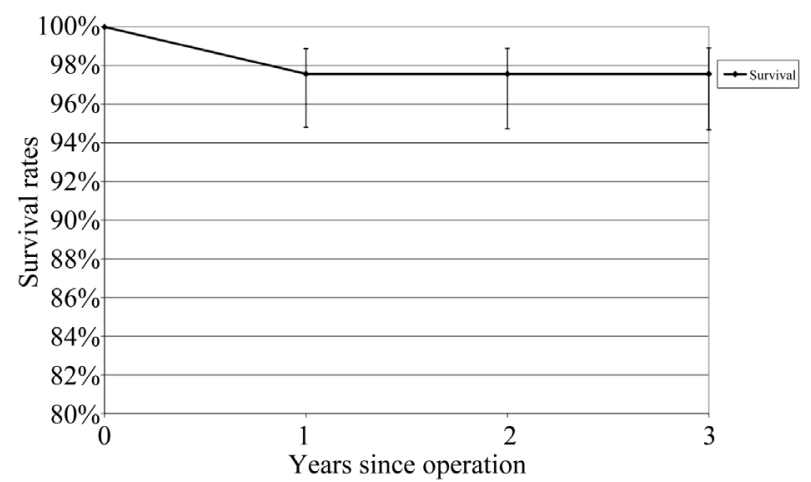

Figure 1. The three-year survival of the PFC Sigma Cobalt Chrome TKA with the endpoint taken as "reoperation for any reason”. Error bars indicate $95 \%$ Confidence Interval.

sening or mechanical failure" (Figure 2). The "worse case" survival was $94.3 \%$ at three years where all the knees lost to follow-up were assumed to have failed.

Radiographic data were available for 219 TKAs $(88.0 \%)$ at final review. Of these 17 radiolucencies were noted in 17 (8\%) cases. The mean mediolateral alignment was 5.2 degrees valgus (2 Degrees Varus to 9 Degrees Valgus.) In this series, 22 (10\%) knees were out-with the suggested range of $7^{\circ}+/-3^{\circ}$ [11]. There was no evidence of increased incidence of radiographic lucency in these knees.

\section{Discussion}

We present the first early clinical, radiological and patient reported results for the PFCSCC TKA. We have previously reported on the medium- to long-term follow up of the PFC Sigma TKA [9-13]. The design of the PFC Sigma Cobalt Chrome TKA incorporates a number of minor modifications from the PFC Sigma TKA and these initial results show that at three years post-operatively, the prosthesis survival rate stands at $97.6 \%$ with revision for any reason and $99.6 \%$ with revision for aseptic failure. This is comparable to our experience with the PFC Sigma where there were a small number of failures, mostly in the early period. This would suggest that the design changes have not caused any increase in early failures.

There are few papers which describe the early results of total knee replacements, however when compared to Munziger et al. 2010 [14], their 5 year results of the Innex total knee replacement demonstrated a 3\% aseptic revision rate and a $4.8 \%$ overall revision rate, whereas our results compare favorably at the moment and show no initial early failures. The Munziger et al. 2010 [14] paper, showed that all their infective revisions were in the first 4 months and after discussing with the authors, 6 out of 9 aseptic revisions were within the 3 years, and these were due to overstuffing, instability anterior knee pain or arthrofibrosis. This paper does confirm the need 


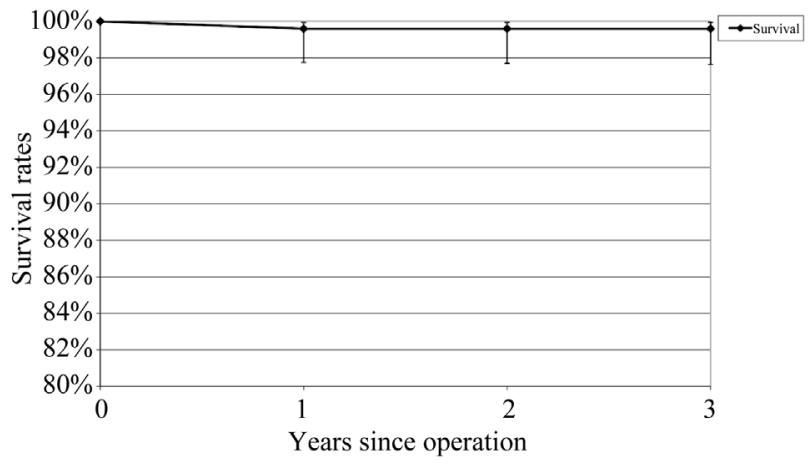

Figure 2. The three-year survival of the PFC sigma cobalt chrome TKA with the endpoint taken as "component revision for aseptic loosening or mechanical failure”. Error bars represent $95 \%$ confidence interval.

to produce five year results to ensure that there is no midterm peak in revisions. Apart from our own results, there have been other medium term results published for the predecessor to the PFCSCC, the PFC Sigma, with Dalury et al. [15] reporting 99.6\% and Zaki et al. [16] reporting $99.4 \%$ survivorship in the medium term and our revisions for aseptic reasons currently remain on track to be similar to their findings. All of our aseptic failures, required a change of the polyethylene spacer only, with none requiring revision of the tibial or femoral components. This comparison is reassuring, following the design modifications made in changing from PFC Sigma to the PFC Sigma Colbalt Chrome, as there appears to be no drop in survival of the prosthesis during the first 3 years.

There were 8 knees (3\%) lost to follow-up during the study period, which highlights the problems with longitudinal studies and represents a weakness in our study. As revision rates are very low, failure to identify a revision from the study group would have a significant effect on the results. If we take a "worst case" scenario, assuming all lost knees were revised, this would give us a survival rate of $94 \%$ at three years. This would still represent an acceptable performance at three-year post implantation.

The clinical outcomes were assessed using the American Knee Society scoring system and the results showed pre-operatively the mean AKS Score was 46.2 whilst at 3 years post-operatively, the mean AKS was 88.3. These early results suggest considerably better outcomes than Zaki et al. 2007 [16], and Hunter et al. 2009 [9], who reported an AKS score at 7 - 9 year review for the PFC Sigma and the result compares well with the Wrightington study $[16,17]$ which had a mean score of 50 and Dalury's results [14] which had a mean score of 50 [15].

The American Knee Society Scoring system is separated into a Knee score and the Function Score to prevent confounding of the Knee Scores by increasing infirmity, and we showed a significant improvement in both Knee Score (mean of 46.2 with a range of 0 - 95 to a mean of 88.3 with a range of $17-100$ at 3 years) and Function Score (mean of 43.5 with a range of $-20-85$ to a mean of 59.5 with a range of -20 - 90 at 3 years). The mean pain score at admission to 3 year review was significantly increased from 11.7 to 39.5. Our results for the PFC sigma ten year results [10] showed a mean function score of 79.9 at 18 months post surgery which then declined to 68.9 at 10 years. Our initial results for the PFCSCC perform favourably when compared to the PFC Sigma, but Arthur et al. 2013 [11] study highlights the importance of continuing to review the AKS for the PFCSCC in order to ensure the scores do not decrease more than expected over time.

The Oxford Knee Score showed a highly significant improvement [16] between the pre-operative score and the scores at 3 months, and the 3-month score and the score at 1 year but there was no significant difference between 1 year and at 3 years, this information is important for patients as it demonstrates that up to one year patients can expect improvements, however these will stabilise out between 1 and 3 years. Our early results were better than the mean Oxford Knee Score for the PFC Sigma at 7 - 9 years [9] and so further research will be required at the ten year mark to ascertain if these early improvements are maintained or diminish.

We used the SF-12 [18] to measure health status and the 3 month review showed significant improvements in all domains except Mental and General Health. After the three month mark the only ongoing significant improvement was an increase in the pain score demonstrating that there was an improvement in pain, which continued up to 1 year whilst these are early results, they are still relevant as early results have been shown to be predictive of overall satisfaction rate by Scott et al. 2010 [19].

Radiographs of the study group were also available for review and 219 knees of the 235 surviving knees were analysed. 22 knees (10\%) were outside the suggested range $\left(7+/-3^{\circ}\right)$ for mediolateral alignment. This figure compares well to other TKA series using the same definition that reported that only $22 \%$ - 34\% lie in this range [20-23]. The standard radiographs taken in our unit are short leg films instead of long leg films, which has the limitation of overestimating varus alignment of the knee by approximately $1.6^{\circ}$. It is likely that our numbers would be lower if long leg films had been used as standard.

\section{Conclusion}

With escalating healthcare costs combined with multiple minor changes to implants, it is essential that we ensure that small changes to the implants are not going to cause early failures in order to prevent multiple costly revisions 
in both monetary and patient morbidity terms. These early results come from the first consecutive cohort of patients using a new implant, with a follow-up rate of $97 \%$ that allows a reliable assessment of the initial efficacy of this prosthesis. These early results should be reproduceble in a district general orthopaedic unit. We conclude that the new PFC Sigma Colbalt Chrome knee arthroplasty performs well over the first 3-year post implantation with excellent survival rates and good clinical and radiological outcomes and that ongoing follow-up is required to ensure that it continues to maintain this standard.

\section{Acknowledgements}

We would like to thank Anne Simpson, Lorraine McCominskie, Shona Mckean, Cheryl Wilson and Janette MacDonald and for their hard work in the data collection in the follow up clinics.

Conflict of interest statement: None of the authors has received or will receive benefits for personal or professional use from a commercial party directly or indirectly related to the subject of this article. However benefits have been received which have been solely directed to a non-profit making research fund with which one or more of the authors are associated.

\section{REFERENCES}

[1] No Authors, "National Joint Registry for England and Wales Summary to the 7th Annual Report," 2009.

[2] No Authors, "Johnson and Johnson. Company Literature and Marketing,” PFC Sigma Cobalt-Chrome, Leeds, 2010.

[3] F. Hinrichs, M. Kuhl, U. Boundroit and P. Griss, "A Comparative Clinical Outcome Evaluation of Smooth (10 13 Year Results) versus Rough Surface Finish (5 - 8 Year Results) in an Otherwise Identically Designed Cemented Titanium Alloy Stem," Archives of Orthopaedic and Trauma Surgery, Vol. 123, No. 6, 2003, pp. 268-272. http://dx.doi.org/10.1007/s00402-003-0515-y

[4] R. Y. Liow and D. W. Murray, "Which Primary Total Knee Replacement? A Review of Currently Available TKR in the United Kingdon," Annals of The Royal College of Surgeons of England, Vol. 79, No. 5, 1997, pp. 335-340

[5] J. N. Install, L. D. Dorr, R. D. Scott and W. N. Scott, "Rationale of the Knee Society Clinical Rating System," Clinical Orthopaedics, Vol. 248, 1999, pp. 13-14.

[6] P. Bennett, "A Comparison of Methods Used for the Prevention of Venous Thromboembolic Disease among Orthopaedic Surgeons at Wolverhampton, United Kingdom and Auckland, New Zealand,” Journal of the Royal Naval Medical Service, Vol. 95, No. 2, 2009, pp. 81-88.

[7] D. W. Murray, A. J. Carr and C. Bulstrode, "Survival Analysis of Joint Replacements," The Journal of Bone \&
Joint Surgery, Vol. 75B, No. 5, 1993, pp. 697-704.

[8] R. D. Ferdinand and I. M. Pinder, "Survival Analysis of Joint Replacements," The Journal of Bone \& Joint Surgery (British), Vol. 79, No. 5, 1997, p. 878.

[9] N. Hunter, R. A. E. Clayton and I. J. Brenkel, "Press Fit Condylar Sigma Total Knee Arthroplasty: 7 - 9 Years Results," European Journal of Orthopaedic Surgery \& Traumatology, Vol. 19, No. 6, 2009, pp. 409-413. http://dx.doi.org/10.1007/s00590-009-0446-6

[10] R. A. E. Clayton, A. K. Amin, M. S. Gaston and I. J. Brenkel, "Five-Year Results of the Sigma Total Knee Arthroplasty,” Knee, Vol. 13, No. 5, 2006, pp. 359-364. http://dx.doi.org/10.1016/j.knee.2006.06.003

[11] C. A. Arthur, A. M. Wood, A. C. M. Keenan, R. A. E. Clayton and I. J. Brenkel, "Ten-Year Results of the Press Fit Condylar Sigma Total Knee Arthroplasty,” BJJ, Vol. 95, No. B2, 2013, pp. 1-4.

[12] A. C. M. Keenan, A. M. Wood, C. A. Arthur, P. J. Jenkins, I. J. Brenkel and P. J. Walmsley, "Ten-Year Survival of Cemented Total Knee Replacements in Patients Aged Less than 55 Years," The Journal of Bone \& Joint Surgery (British), Vol. 94B, No. 7, 2012, pp. 928-931. http://dx.doi.org/10.1302/0301-620X.94B7.27031

[13] A. M. Wood, A. C. M. Keenan, C. H. C. Arthur, S. A. Aitken, P. J. Walmsley and I. J. Brenkel, "The Functional Outcome of Total Knee Replacement in Young Patients: A 10-Year Matched Case Control Study," OJO, Vol. 3, No. 2, 2013, pp. 128-132.

[14] U. K. Munzinger, N. A. Maffiuletti, T. Guggi, M. Bizzini, S. Preiss and T. Drobny, "Five-Year Results of the Innex Total Knee Arthroplasty System," International Orthopaedics, Vol. 34, No. 8, 2010, pp. 1159-1165. http://dx.doi.org/10.1007/s00264-009-0887-x

[15] D. F. Dalury, R. A. Gonzales, M. J. Adams, T. A. Gruen and K. Trier, "Midterm Results with the PFC Sigma Total Knee Arthroplasty System," The Journal of Arthroplasty, Vol. 23, No. 2, 2008, pp. 175-181. http://dx.doi.org/10.1016/j.arth.2007.03.039

[16] S. H. Zaki, I. Rafiq, A. Kapoor, R. Videsh, A. K. Gambhir and M. L. Porter, "Medium Term Results with the Press Fit Condylar (PFC) Sigma Knee Proisthesis the Wrightington Experience," Acta Orthopaedica Belgica, Vol. 73, No. 1, 2007, pp. 55-59.

[17] S. L. Whitehouse, A. W. Blom, A. H. Taylor, G. T. R. Pattison and G. C. Bannister, "The Oxford Knee Score; Problems and Pitfalls,” The Knee, Vol. 12, No. 4, 2005, pp. 287-291. http://dx.doi.org/10.1016/j.knee.2004.11.005

[18] D. L. Riddle, K. T. Lee and P. W. Stratford, "Use of SF-36 and SF-12 Health Status Measures: A Quantitative Comparison for Groups versus Individual Patients," Medical Care, Vol. 39, No. 8, 2001, pp. 867-878. http://dx.doi.org/10.1097/00005650-200108000-00012

[19] C. E. H. Scott, C. R. Howie, D. MacDonald and L. C. Biant, "Predicting Dissatisfaction Following Total Knee Replacement," Journal Bone and Joint Surgery (British), Vol. 92, No. B9, 2010, pp. 1253-1258. http://dx.doi.org/10.1302/0301-620X.92B9.24394

[20] H. Bathis, L. Perlick, M. Tingart, C. Luring, D. Zura- 
kowski and J. Grifka, “Alignment in Total Knee Arthroplasty. A Comparison of Computer-Assisted Surgery with the Conventional Technique," Journal Bone and Joint Surgery (British), Vol. 86B, No. 5, 2004, pp. 682-687

[21] M. J. Bankes, D. L. Back, S. R. Cannon and T. W. Briggs, "The Effect of Component Malalignment on the Clinical and Radiological Outcome of the Kinemax Total Knee Replacement,” Knee, Vol. 10, No. 1, 2003, pp. 55-60. http://dx.doi.org/10.1016/S0968-0160(02)00050-9

[22] A. Harvey, M. P. Manning, S. A. Sampath, R. Johnson and M. A. Elloy, "Alignment of Total Knee Arthroplasty: the Relationship to Radiolucency around the Tibial Component,” Medical Engineering \& Physics, Vol. 17, No. 3, 1995, pp. 182-187.

http://dx.doi.org/10.1016/1350-4533(95)95708-I

[23] J. Mahaluxmivala, M. J. Bamkes, P. Nicolai, C. H. Aldam and P. W. Allen, "The Effect of Surgeon Experience on Component Position in 673 Press Fit Condylar Posterior Sacrificing Total Knee Arthroplasties," The Journal of Arthroplasty, Vol. 16, 2001, pp. 635-640. http://dx.doi.org/10.1054/arth.2001.23569 